\title{
Broadband amplitude-stabilized oscillator
}

\author{
JULIUS FOIT \\ Department of Microelectronics \\ CTU Prague \\ Technická 12, 16627 Praha 6 \\ Czech Republic
}

\begin{abstract}
In many applications we need oscillators tunable over a considerable frequency range by simple LC circuits with broadly varying dynamic resistances (quality factor $\mathrm{Q}$ of the reactances), while insisting on keeping a rather constant amplitude of the A.C. voltage across the LC circuit, or at least of some output voltage (not necessarily harmonic waveform) somewhere in the circuit. If we manage to arrange the circuit in such a way that a variable external load will not cause appreciable frequency shifts of the oscillations, it will be felt as a special benefit. Another special benefit will be welcomed if the circuit does not require any taps (inductive or capacitive) or any transformer coupling in the frequency-determining LC circuit. Perhaps even more appreciated will be a possibility to have one side of the LC circuit grounded. The following paper shows a surprisingly simple circuit capable to fulfill these seemingly conflicting requirements, all at he same time.
\end{abstract}

Key-Words: - oscillators, distortion, waveform, LC tuning, stable amplitude

\section{Requirements}

The requirements specified above indicate that we need a circuit possessing adequate internal automatic adjustment of the basic oscillation condition - the loop gain. The circuit must have sufficient reserve of gain in order to oscillate with poor-Q LC circuits, and at the same time have enough regulation capability to keep the amplitude of LC circuit oscillation voltage within a rather narrow tolerance band. Keeping the oscillation amplitude within reasonable limits also improves frequency stability of the oscillator since it keeps the THD (total harmonic distortion) of the generated waveform relatively low. Theoretically there exist several methods for obtaining such behavior of oscillator circuits; unfortunately, however, most of them are rather complex and not at all suited for LC tuning in a broad frequency range. Yet it can be shown that making use of the basic properties of semiconductor active devices, it is possible to obtain quite acceptable behavior by a rather simple circuit.

\section{Basic principle}

An example of one of the well-known basic arrangements of an LC oscillator is shown in Fig. 1. The amplifier in this case is operating as a noninverting voltage-controlled current source. The LC circuit serves as a converter, converting the output

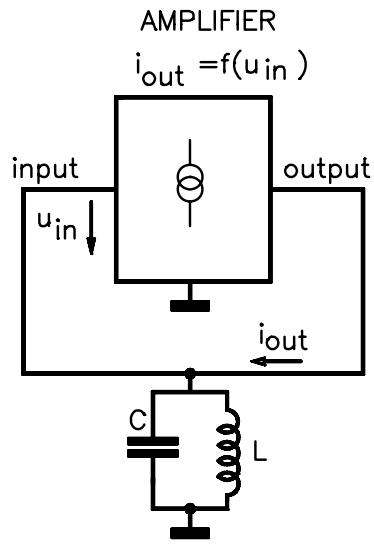

Fig. 1 Oscillator principle

$\ell_{\text {in }}$, serving as input to the amplifier. The oscillation condition can be formally written as

$$
A_{o}={ }_{u_{\text {in }}}^{i_{\text {in }} R_{d}} \geq 1
$$

where

$A_{O}$ is the overall voltage amplification,

$R_{d}$ is the dynamic resistance of the LC circuit at its resonant frequency. 
Of course, in real circuits, the value of $R_{d}$ depends on the particular properties of the LC circuit and can lie anywhere within a range of several orders of magnitude. Also, equation (1) assumes that the amplifier itself is ideal, i.e. frequency-independent which frequently is not true. Nevertheless, Fig. 1 and equation (1) give a simple insight in the basic problem: in order to secure proper operation: the amplifier properties must be adjusted in a wide range if we assume that very different LC circuits are going to be used. It is certainly possible to adjust the amplification so that the condition (1) is securely fulfilled for the worst-case LC circuit and just rely on the fact that excessive amplification will finally be cut down by nonlinearities in the amplifier under conditions of severe overdrive. This, however, is far from the optimum oscillator operating conditions. A heavily overdriven amplifier frequently loses most of its good properties. First of all, its input and output differential resistance can drop to a fraction of the optimum (high) value. Second, a large degree of nonlinear distortion can appear in the waveform of the current driving the LC circuit, impairing frequency stability. Moreover, all these effects are strongly dependent on the momentary value of the amplifier power supply voltage, causing a further deterioration of frequency stability.

\section{$3 \quad$ Solution}

Different kinds of real LC oscillator circuits use very different internal design structures of the amplifier block (Fig. 1). The popular common emitter or common source transistor stage has two important drawbacks: it is an inverting amplifier, and its output does not really behave as a good current source,

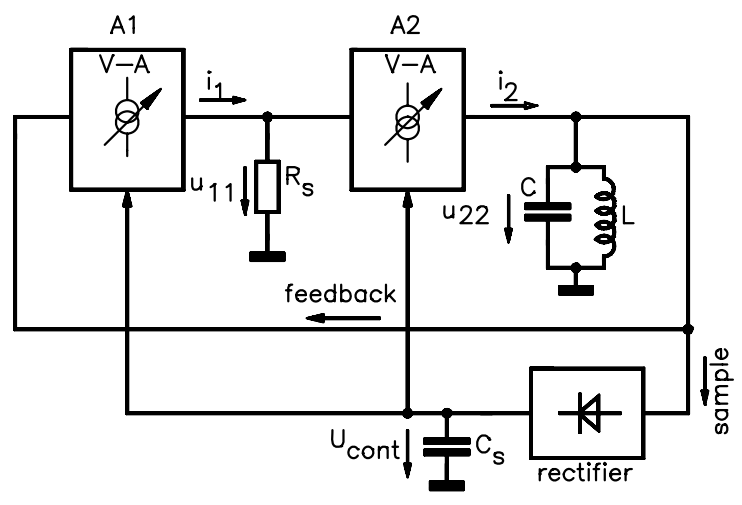

Fig. 3 Oscillator with automatic gain control

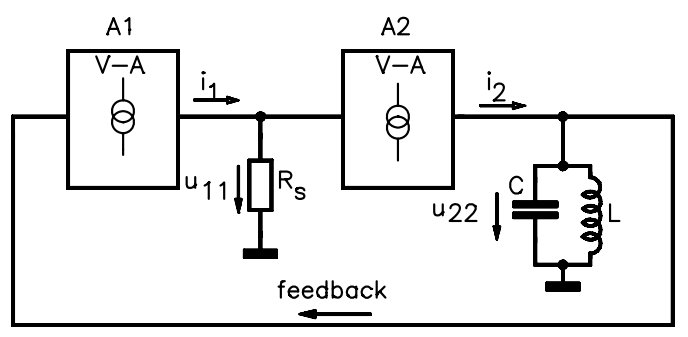

Fig. 2 Two-stage LC oscillator

especially in the conditions of heavy overdrive. Various well-known oscillator circuits try to avoid these problems either by transformer coupling or by taps on the LC circuit. Both methods complicate the LC circuit design and remove the problems only partially. Moreover, this solution does not at all address the wide variability of LC circuit parameters appearing in real life; as a result, oscillators with tapped or transformer-coupled LC circuits must be individually designed for each particular LC circuit in order to obtain optimum operating conditions.

We will show in the following that most of the problems mentioned above can be solved in a very efficient way by choosing a cascaded pair of two amplifier stages, both non-inverting and operating as voltage-to-current converters, instead of a single amplifier stage. The general idea of the circuit is shown in Fig. 1. It consists of two amplifying stages $A 1, A 2$, each operating as a non-inverting voltage to current converter (voltage controlled current source). The output current $i_{1}$ of stage $A l$ is converted to voltage $u_{11}$ by passing it through the coupling resistor $R_{s}$. The $u_{11}$ voltage serves to drive the second stage $A 2$. The output current of stage $A 2$ is converted to voltage once again, but this time the output voltage $u_{22}$ is generated over the dynamic resistance of a LC tuned circuit . The

voltage $u_{22}$ is fed back to the input of stage $A 1$, completing the positive-feedback loop. The over all loop amplification can be written as

$$
A_{t o t}=A 1 \cdot A 2=R_{s} \cdot R_{d} \cdot Q \cdot \omega \cdot L \cdot\left|y_{21 s 1}\right| \cdot\left|y_{21 s 2}\right|
$$

where

$R_{d} \quad$ is the dynamic resistance of the LC circuit at resonance (at the $\omega$ frequency),

$Q \quad$ is the quality factor of the LC circuit,

$A 1, A 2$ are the equivalent voltage amplifications of both amplifier stages, 
$\left|y_{21 s}\right|,\left|y_{21 s 2}\right| \quad$ are the real parts of differential transfer admittances of both amplifying stages.

The basic condition (1) for self-sustained oscillations, $A_{\text {tot }}>1$ must be fulfilled for all expected $R_{d}$ values of the LC circuit involved. Theoretically, the fulfillment of this condition presents no problem; a totally different situation, however, appears in real life when we insist on having the circuit operating as an LC oscillator with

a) a broad range of tuning inductances and capacitances (several orders of magnitude for each of them),

b) a broad range of quality factors $Q$ of the LC circuit (mostly determined by the inductor),

c) obtaining the same signal amplitude from the oscillator at any of a) and b) conditions, and

d) at the same time, keeping the best possible frequency stability versus supply voltage and load.

Most of the common simple LC oscillator circuits are just unable to fulfill all these requirements at the same time. Indeed there do exist oscillator circuits capable of fulfilling some of the mentioned requirements one by one, but none of them is able to fulfill all of them without raising the circuit complexity beyond reasonable limits. If we can arrange the voltage-tocurrent converting stages $A 1, A 2$ in such a way that their conversion coefficients (i.e. amplification) can be controlled by an external D.C. signal, and add a circuit deriving such a control signal from the $u_{22}$ voltage amplitude, the block diagram of Fig. 2 will change to Fig. 3. Here, the symbols of current sources in the amplifier blocks were supplemented with "control" arrows to stress the fact that the amplification can be electronically controlled from outside. When the amplification control is not applied to just one stage but rather to both of them, its efficiency is increased considerably. In fact, we have added one more feedback to the oscillator circuit in addition to the original positive feedback needed for oscillations, this time a negative one, limiting the amplitude of output voltage $u_{22}$ across the LC circuit.

Now, let us try to find the best possible active devices to be used in the amplifier blocks $A 1, A 2$. For best operation they should fulfill five basic requirements:

- their A.C inputs should be. voltage-controlled

- the amplification should best be a linear (or inverse) function of the D.C. control voltage
- $\quad$ the output of $A 2$ should have the highest possible differential internal resistance (current source)

- both stages should be non-inverting

- input currents of both stages should be as small as possible (preferably zero).

The result of these deliberations is clear: the best active devices for both amplifier stages are JFETs, $A 1$ operating as a common drain amplifier, $A 2$ operating in common gate connection. The resulting final circuit schematic diagram is shown in Fig. 5. Type BF245B JFETs were chosen for both stages, selected units with drain current $5 \mathrm{~mA}$ at $\mathrm{V}_{\mathrm{GS}}=0$ and at $\mathrm{V}_{\mathrm{DS}}=15 \mathrm{~V}$.

As can be seen from Fig. 5, transistor $Q 1$ operates in common drain connection as amplifier stage $A 1$, transistor $Q 2$ as amplifier stage $A 2$. The rectification

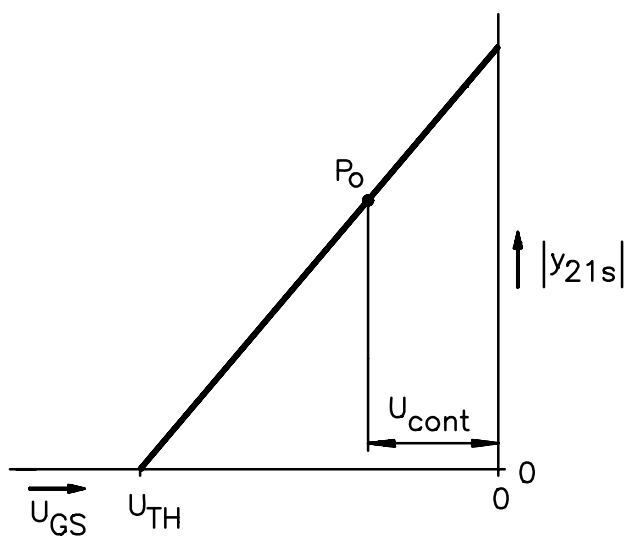

Fig. 4 Control characteristic of a JFET

of LC circuit A.C. voltage "sample" (see Fig. 4) is performed by the gate junction of Q1. The coupling capacitor $\mathrm{C} 4$ serves also as the smoothing capacitor $\mathrm{C}_{\mathrm{S}}$ (see Fig. 4) and the D.C. control voltage is brought to the gate of $Q 2$ through the resistor R2. C2 connects the gate of $Q 2$ to the common conductor for A.C. signal components, i.e. to operate $Q 2$ in common gate connection, driven into its source from the source of $Q 1$. A rather small-value resistor $\mathrm{R} 4$ is added in series with the drain of $Q 2$ to provide an output waveform well insulated from the frequency-determining components of the circuit. In this way, the effects of variations of load at the output terminals on the operating frequency are minimized. Not negligible advantages of the circuit in Fig. 5 are the facts that one side of the LC circuit is directly connected to the common conductor (grounded) and that no capacitive or inductive tapping or transformer coupling is needed in the tuning circuit, just a plain parallel LC combination. 


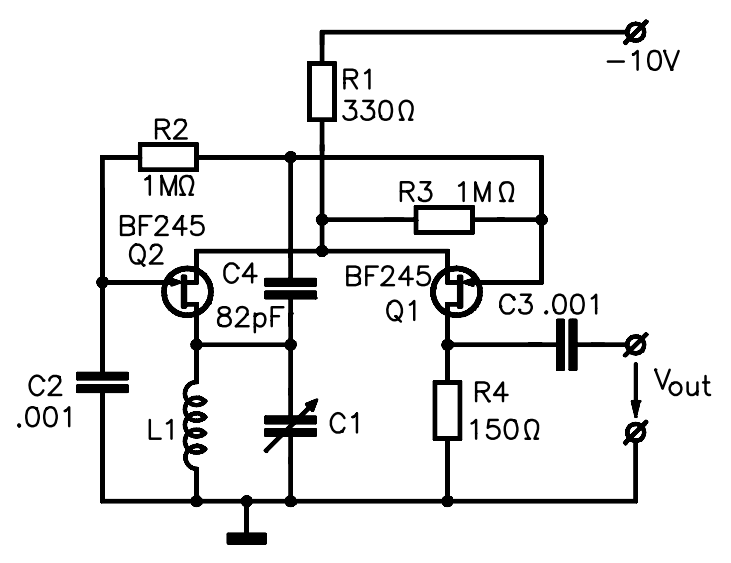

Fig. 5 Final oscillator circuit

\section{$4 \quad$ Results}

The oscillograms in Fig. 6 and Fig. 7 show that no appreciable changes in output amplitude appear even when widely different LC circuits are used; as a matter of fact, the same output voltage is obtained even at frequencies as low as $5 \mathrm{kHz}$ and as high as $50 \mathrm{MHz}$ with no adjustment necessary of any passive component values of the circuit (except the L1, C1 values, of course). The amplitude shown in Figs 6 and 7 remains constant within 3\% for supply voltages from 8 to 30V [1]. The same or better amplitude stability holds for the output voltage $V_{\text {out }}\left(1.8 \mathrm{~V}_{\mathrm{P}-\mathrm{P}}\right.$, half-sine pulse, in the Fig. 5 circuit).

In certain applications we may need to have the output waveform of the circuit spectrally clean, i.e. a pure harmonic (sine) wave, and at the same time to keep the influence of variable load impedance as low as possible. In such a case we must add a buffer circuit. A very efficient buffer fulfilling these requirements can be built using a similar circuit arrangement as the

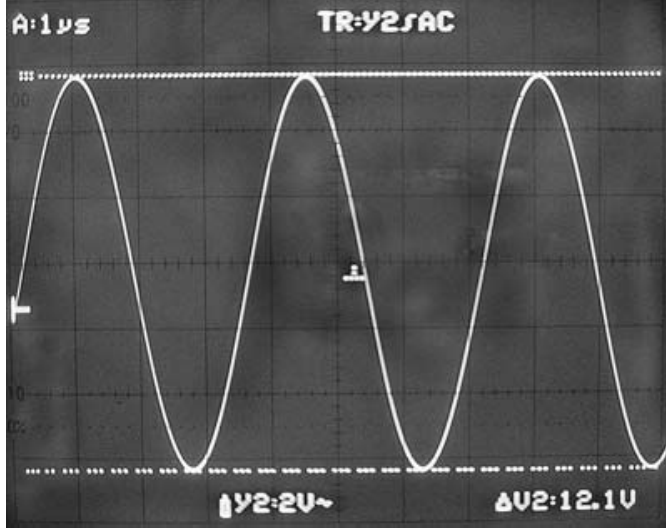

Fig. 6 Output $\mathrm{V}_{\mathrm{LC}}$ waveform at $280 \mathrm{kHz}$.

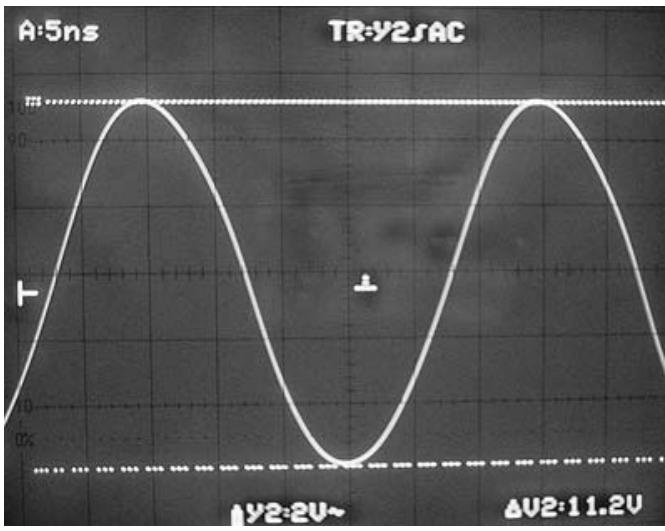

Fig. 7 Output $\mathrm{V}_{\mathrm{LC}}$ waveform at $30 \mathrm{MHz}$. $L 1=0,56 \mu H, C 1=60 p F$

oscillator itself - two JFETs operating as a two-stage source-coupled amplifier. The circuit arrangement is shown in Fig. 8. The extremely small value of the JFET input admittance makes possible to use a very small coupling capacitance (see C2 in Fig. 8), thereby keeping the overall tuning capacitance of the LC circuit essentially concentrated in C1 alone. C3, together with C2, forms a voltage divider which keeps the input voltage low enough to avoid nonlinear

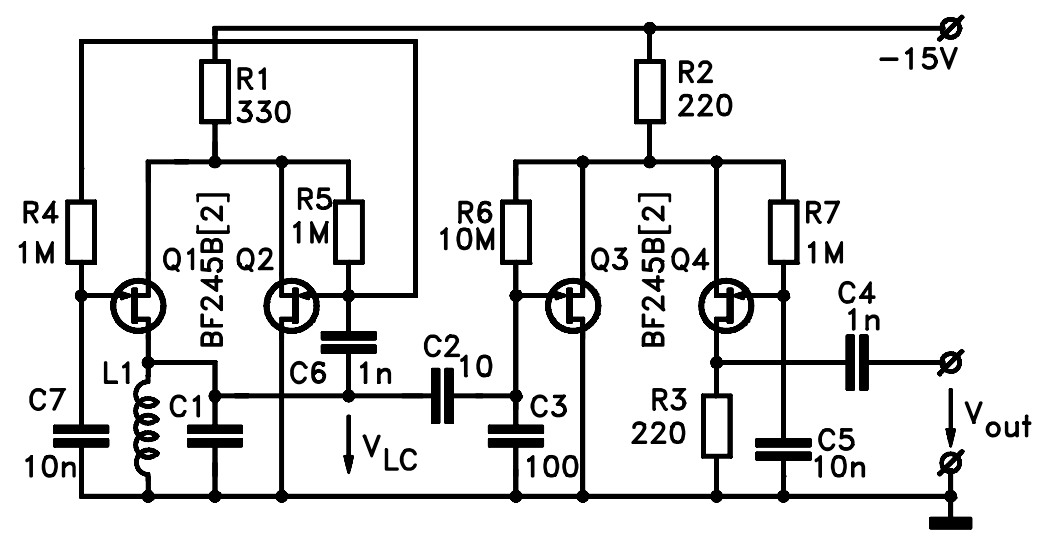

Fig. 8 Oscillator with linear output buffer 
distortion in the first stage of the buffer amplifier (Q3), and further decreases the already small effect of load variations reverse transfer through the buffer amplifier. The buffer output voltage amplitude can be controlled (within certain limits) by the value of R3. The value shown in Fig. 8 yields an overall buffer voltage amplification approximately unity, providing an undistorted harmonic output voltage $\mathrm{V}_{\text {out }} \approx 1.3 \mathrm{~V}_{\mathrm{p}-\mathrm{p}}$.

\section{References:}

[1] Julius Foit, LC oscillator has stable amplitude.

Electronic Design News, Vol. 50 (2005), No. 10, pp.

93 - 96, Reed Elsevier Electronics Group, USA,

ISSN 0012-7515 